\title{
Performance Comparison of WiMAX and WLAN Technologies using OPNET Modeler
}

\author{
Ali GEZER ${ }^{1}$, Marwa Khaleel ${ }^{2}$ \\ ${ }^{1}$ Erciyes University, Kayseri Vocational College, Electronic and Telecommunication Technology, \\ aligezerr@hotmail.com \\ ${ }^{2}$ Erciyes University, Graduate School of Natural and Applied Sciences, marwakhaleel30@yahoo.com
}

\begin{abstract}
Wireless Local Area Network (WLAN) is based on the IEEE 802.11 standard. It is also known as Wireless Fidelity network (Wi-Fi). WLAN is used in small areas such as buildings, homes and campus. WiMAX (Worldwide Interoperability for Microwave Access) is based on IEEE 802.16 standard. WiMAX is like Wi-Fi in use but it covers vast areas like an entire city. In this paper, we compare the performance of WiMAX and WLAN networks for voice, video conferencing, ftp and web applications using OPNET simulator in terms of throughput, delay, jitter. Quality of service (QoS) support for both technologies provide smooth and steady voice and video transfer, controlled jitter and delay and improve loss characteristics. The simulation results show that WiMAX network provides better throughput and load in comparison to WLAN networks. In the case of delay, WLAN gives good result in comparison to WiMAX scenario.
\end{abstract}

Keywords: WLAN, WiMAX, Mobile, QoS, Performance Analysis.

\section{Introduction}

The Internet has a great importance to computer users. It is like a window opening to all over the world. Nowadays, there are many methods for broadband Internet connection like xDSL, cable modem, FTTX, WLAN and WiMAX. Wireless solutions have increased its attraction for the end user due to their increasing bandwith opportunities. WLAN has become one of the most promising and successful technology in recent years. Despite the many advantages of WLAN, WiMAX which is also called Wireless Metropolitan Area Network (WMAN) is a good alternative for mobile internet connections. WiMAX has a very high performance and speed. It can operate in a range up to 40 miles at $70 \mathrm{Mbps}$ while Wi-Fi has a range of running a few hundred feet at speeds up to $54 \mathrm{Mbps}[1,3]$. Mobile WiMAX is considered as a candidate for fourth generation (4G) in competition with the LTE Advanced standard [2].

In this study, we use two scenarios to conduct a comparative performance analysis of WLAN and WiMAX technologies for a small network. We use wireless servers for four applications (web, voip, video and ftp) and operate the networks with quality of service support. We also change the physical layer settings of the devices used, which is explained later in the network design part. This lead to a difference in simulation results for voice, video, traffic sent and received packets..

\subsection{Wi-Fi and WiMAX Overview}

\section{- Wi-Fi}

Wireless Fidelity network (Wi-Fi) has been invented and developed for LAN communication without using cables. Initially, the goal was to serve mobile devices and PCs, but the rapid development of this technology has 
become serving the surfers of the Internet world, especially in cafes, restaurants, hotels, airports and banks where often provided free of charge internet connections to attract customers. Now, Wi-Fi provides great service in sensitive places such as hospital and security sites so that the doctor or security man can access certain applications to serve patients or identify undesirable persons from entering sensitive places. It is also added in smart phones to provide internet access via available Wi-Fi network. Another important feature of these networks is that they can be installed in places where it is difficult to extend cables. Wi-Fi requires a certain number of reinforcement points to cover large area. The network cannot be used outside the coverage areas noting that the coverage of these networks ranges from 32 meters at home and 95 meters in the outside and these numbers can be increased in case of using booster.

\section{- WiMAX}

The aim of World Wide Interoperability for Microwave Access (WiMAX) is to provide long distance communication and link between multiple sites without using cables. The most common applications that use this technology are mobile phones which mainly depend on IP telephone and Internet access service. WiMAX has been used to reconnect quickly between sites when natural disasters occur such as tsunami that hit many countries in Southeast Asia, especially Indonesia. The same situation was repeated in Hurricane Katrina in America, where communication between these affected areas and the rest of the world was lost at that time. Fixed WiMAX operates in 2.5 and $3.5 \mathrm{GHz}$ frequency band, mobile WiMAX operates between 2 and $6 \mathrm{GHz}$. WiMAX reaches the distances more than $45 \mathrm{~km}$ and also the speed $40 \mathrm{Mbps}$ in the case of direct visual vision [4]. WiMAX technology has been deployed in many countries for better service and higher speed at a lower cost. Furthermore, WiMAX is used in many applications including digital control applications to take advantage of the high capacity and achieve high efficiency.

It should be noted that the applications which use two technologies (Wi-Fi and WiMAX) have been increasing as they were initially focused on the mobile and PC and then developed for many of the devices. These technologies are continuously increasing and the future is very promising for them as they are easy to install and clean for the environment.

\subsection{Network Design}

OPNET Modeler 14.5 is used to simulate both of the network technology which consists of two scenarios WiMAX and WLAN.

In the first scenario, WiMAX network model consists of one WiMAX router, 4 base stations, 4 servers and 4 mobile stations for each of the web, video, ftp and VoIP applications. WiMAX configuration node is used for MAC service class definitions.

In the second scenario, WLAN network model consists of similar end points but WiMAX base stations are replaced with wireless routers and WiMAX configuration attribute isn't used.

For both of the scenarios, PPP advanced links are used to connect all the routers and base stations to IP cloud. Weighted Fair Queuing (WFQ) based service class from QoS scheme is implemented for both scenarios. Mobility configuration is necessary for the movement of mobile hosts in any direction which is defined by mobile trajectory attribute.

Application definition attribute and profile definition attribute are used for defining Web, Video and VoIP applications to send the traffic from associated client to its server. Three applications (web, video and VoIP) are assigned to the hosts in our simulation by using applications attribute. The VoIP application uses G.711 encoder scheme and VoIP server service is used for establishing the VoIP calls. The VoIP application requires some guarantee to perform its service with minimum delay and low packet loss. 


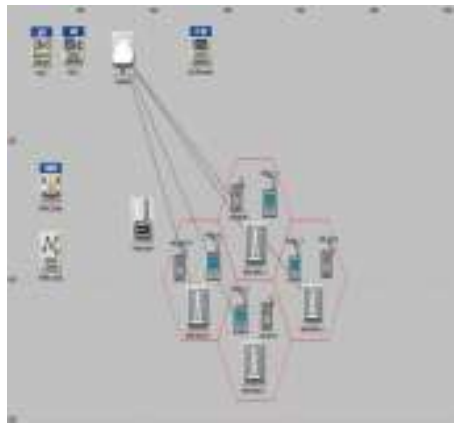

Fig. 1: Scenario 1.WIMAX network Topology

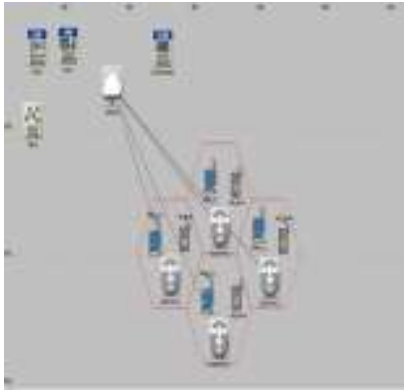

Fig. 2: Scenario 2.WLAN network Topology

\subsection{NetworkParameters}

WiMAX and WLAN parameters used in thenetwork model are presented in Tables below:

TABLE I: Wireless LAN parameters for WLAN Scenario.

\begin{tabular}{cc}
\hline \hline BSS identifier for Access Point & $1,2,3,4$ \\
$1,2,3,4$ & Enabled \\
Access Point Functionality & Direct Squence \\
Physical Characteristics & $11 \mathrm{Mbps}$ \\
Data Rate (bps) & 0.005 \\
Transmit Power (W) & -95 \\
Packet Reception-Power Threshold & 7 \\
Short Retry Limit & 4 \\
Long Retry Limit & 256,000 \\
Buffer Size (bits) & \\
\hline \hline
\end{tabular}

TABLE II: Base Station,router,server,mobile parameters for WiMAX scenarios.

Traffic characteristics

Match property

Match condition

Match value

Service Class Name

WiMAX parameters

Antenna gain (dBi)

MAC address

Maximum transmission power (W)

PHY profile

PermBase

BS parameter

Maximum number of SS nodes

Minimum power density

Maximum power density

Number of initial ranging codes

Number of HO ranging codes

Number of periodic ranging codes

Number of bandwidth request codes

Number of transmitters
IP ToS

Equals

Any

Silver

$15 \mathrm{dBi}$

Auto Assigned

0.5

Wireless OFDMA $20 \mathrm{MHz}$

0

100

$-100$

$-60$

8

8

8

8 
SS parameter

-Downlink Service Flow/-Uplink Service Flow

Service Class Name

Modulation and Coding

Silver

Average SDU Size (bytes)

64-QAM 1/2

Activity Idle Timer (seconds)

1500

Buffer Size (bytes)

\section{Simulation Results}

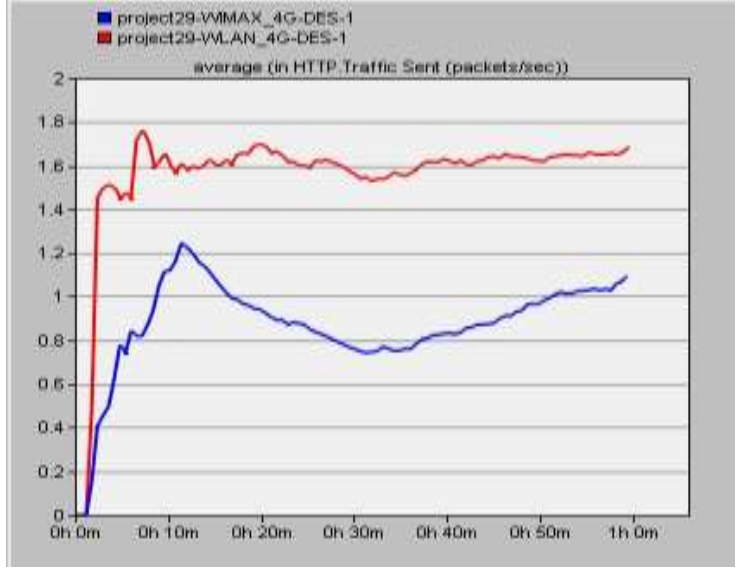

(a)

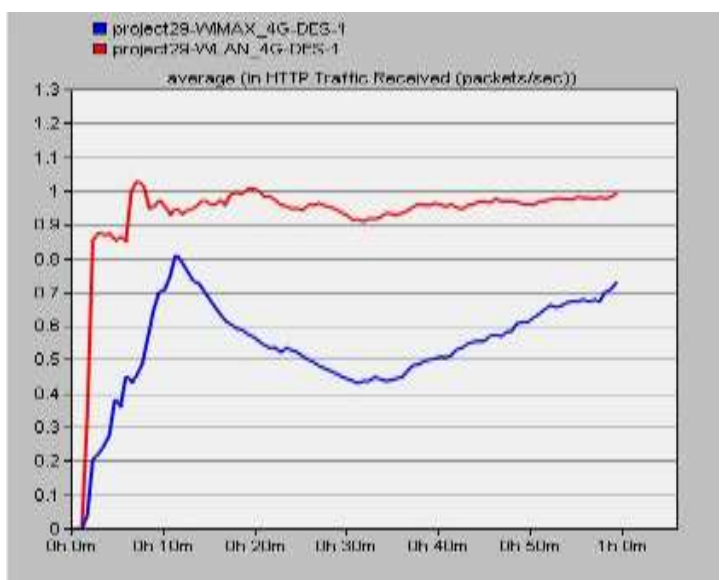

(b)

Fig.3:HTTP Traffic Sent and Received

HTTP sent and received packets are shown in Figure 3. Approximately, the ratio between sent and received packets in second are preserved for both technologies. But WLAN performs higher traffic sent and receive rate in comparison to WiMAX technology.

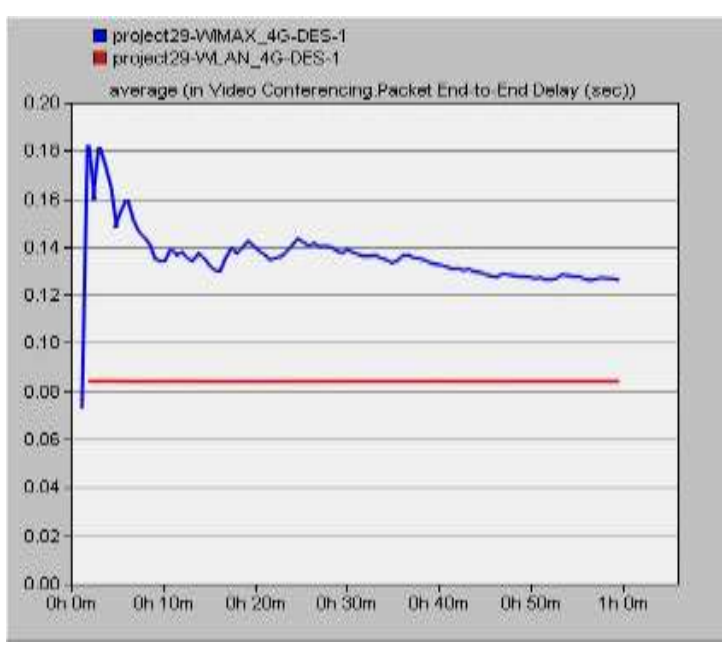

Fig. 4: Video end to end delay

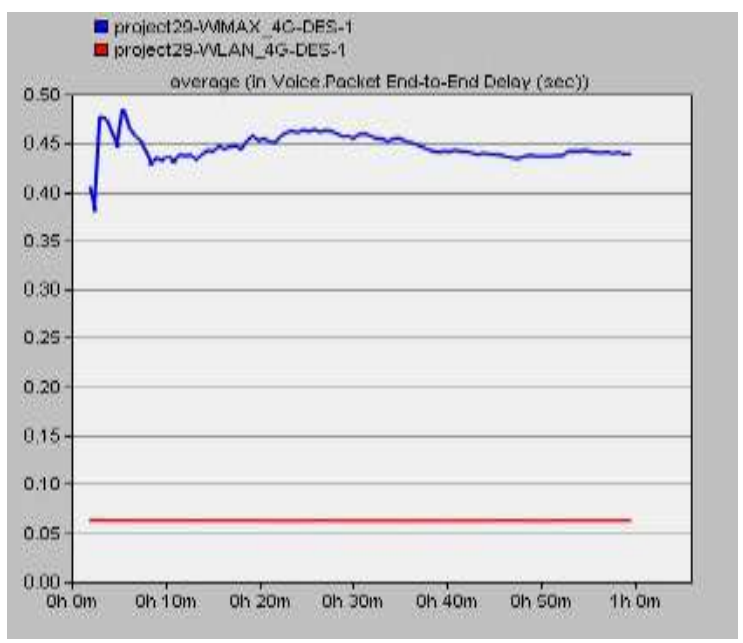

Fig. 5: Voice end to end delay 
Fig. 4 explains the same quantity for Video application .Fig. 5 shows average packet end to end delay in seconds. WiMAX gives nearly 0.48 seconds and WLAN gives nearly 0.06 seconds for voice traffic. In both applications, WLAN gives good results in terms of packet end to end delay.

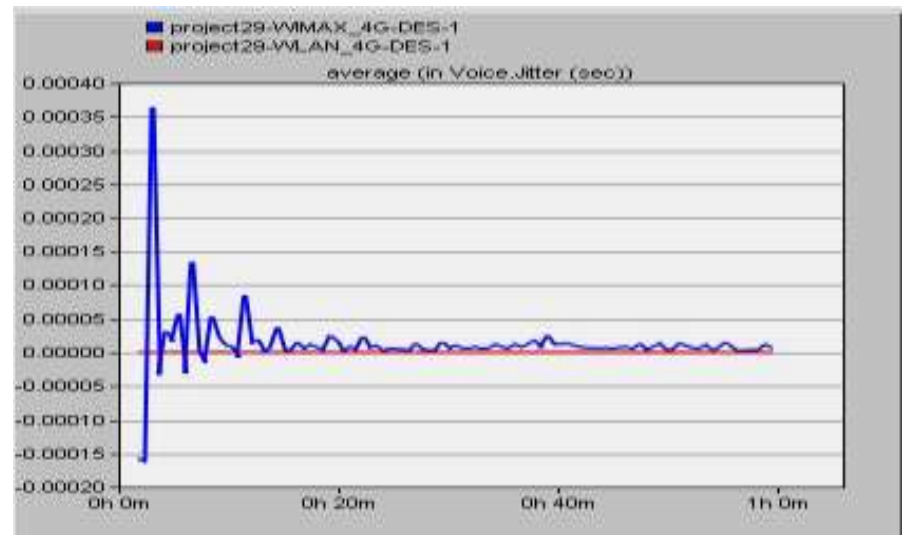

Fig. 6:Voice jitter

Fig, 6 shows voicejitter in both network models. Via the simulation results, we observe thatWiMAX jitter is higher as compared to WLAN Jitter.

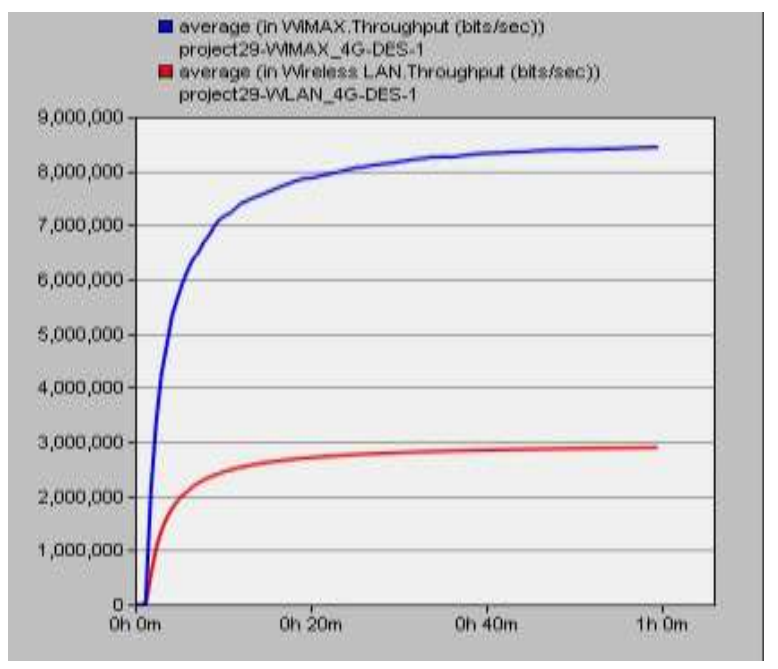

Fig.7: Throughput

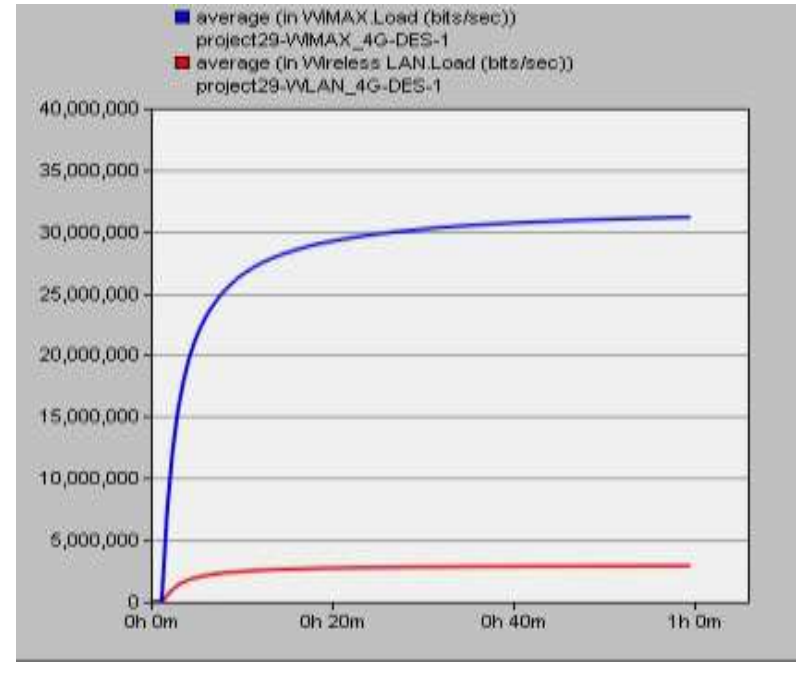

Fig. 8: Load

In terms of throughput and traffic load, WiMAX is superior than WLAN technology according to the simulation results as shown in Figure 7 and Figure 8.

\section{Conclusion}

The primary goal of this research is to compare the performances of the WLAN and WiMAX technologies in terms of web, video conference, ftp and VoIP application traffic through OPNET program. Both technologies are replacing wired solutions such as Ethernet technologies with their increasing bandwith opportunities. After viewing the simulation results we can conclude that WiMAX network gives better results in throughput, traffic load WLAN gives good results for the parameter end to end delay and voice jitter. 


\section{References}

[1] R. Paul, S. Lally and Lj. Trajkovic,"Simulation and performance evaluation of WiFi and WiMAX using OPNET”,Opnetwork 2011, Washington, DC, 2011.Communicatipon Networks SPRING 2011.

[2] N. Bagoria, A. Garhwal, A. Sharma" Simulation of Physical layer of WiMAX Networkusing OPNET Modeller", International Journal of P2P Network Trends and Technology (IJPTT), Vol.3 Iss.4, May 2013.

[3] G. Malik, A. Singh"Performance Evaluation of WiFi and WiMax Using Opnet",International Journal of Advanced Research in Computer Science and Software Engineering, Volume 3, Issue 6, 2013.

[4] J. M. Sultan, G. Markarian and P. Benachour'Integration of WiFi and WiMAX Services: Bandwidth Optimization and Traffic Combination", 2015 DLINE. 